\title{
Subtotal Parathyroidectomy for Tertiary Hyperparathyroidism: a Case Report and Literature Review
}

\author{
Younil Jang ${ }^{1}$, Gheun-Ho Kim², Jung Hwan Park ${ }^{2}$ and Kyung Tae \\ Departments of Otolaryngology-Head and Neck Surgery ${ }^{1}$ and Internal Medicine ${ }^{2}$, College of Medicine, Hanyang University, Seoul, \\ Korea
}

Despite the correction of secondary renal hyperparathyroidism after successful kidney transplantation, some recipients have persistent hyperparathyroidism due to autonomous hypertrophied parathyroid glands. St. Goar first identified and termed this disease as tertiary hyperparathyroidism. Surgery, either subtotal parathyroidectomy or total parathyroidectomy with autotransplantation, is the main treatment for tertiary hyperparathyroidism. Here, we report a case of a patient with tertiary hyperparathyroidism after two times of kidney transplantation who underwent subtotal parathyroidectomy and also review the relevant literature.

Key Words: Hyperparathyroidism, Kidney transplantation, Parathyroidectomy

\section{Introduction}

Approximately $90 \%$ of patients with chronic kidney disease (CKD) develop secondary hyperparathyroidism by the time maintenance hemodialysis is initiated. ${ }^{1)}$ The pathogenesis of renal hyperparathyroidism is complex and not yet completely understood. Hyperphosphatemia, decreased 1,25-dihydroxyvitamin D level, and the resultant slight decrease in serum calcium level are considered the main contributors to the pathogenesis of renal hyperparathyroidism. Abnormalities in the renal tubular absorption of phosphate lead to reduced phosphate excretion and hyperphosphatemia. Impaired renal conversion of 25-hydroxycholecalciferol to 1,25-dihydroxycholecalciferol (vitamin D, calcitriol) cause a decrease in the intestinal absorption of calcium. As a consequence of prolonged hypocalcemia, parathyroid chief cell hyperplasia occurs and parathyroid hormone (PTH) secretion increases. ${ }^{2)}$

After successful renal transplantation, renal tubular function and renal conversion of vitamin $D$ are restored. Therefore, hypertrophied parathyroid tissue resolves and PTH secretion normalizes. However, despite the correction of their chronic kidney disease, about one third of kidney recipients have high PTH level during 6 months and high PTH concentration continues for five years in $20 \%$ of kidney recipients due to autonomous hypertrophied parathyroid glands. ${ }^{3)}$ St. Goar recognized this disease, terming it "tertiary hyperparathyroidism". ") The management of tertiary hyperparathyroidism remains controversial. In the majority of patients with tertiary hyperparathyroidism after kidney transplantation, hypercalcemia and hypophosphatemia usually resolve within the first year. Patients with persist hypercalcemia lasting one year or more after kidney transplantation should consider parathyroidectomy. Calcimimetic agent such as cina-

Received October 15, 2018 / Revised May 22, 2019 / Accepted July 30, 2019

Correspondence: Kyung Tae, MD, PhD, Department of Otolaryngology-Head and Neck Surgery, College of Medicine, Hanyang University, 222 Wangsimni-ro, Seongdong-gu, Seoul 04763, Korea

Tel: 82-2-2290-8585, Fax: 82-2-2293-3335, E-mail: kytae@hanyang.ac.kr

Copyright (c) 2019, the Korean Thyroid Association. All rights reserved

() This is an open-access article distributed under the terms of the Creative Commons Attribution Non-Commercial License (http://creativecommons.org/licenses/by-nc/4.0/), which permits unrestricted non-commercial use, distribution, and reproduction in any medium, provided the original work is properly cited. 
calcet exhibits allosteric modulation of the calcium receptor on the parathyroid gland, and it may serve as an alternative to parathyroidectomy for treatment of renal or tertiary hyperparathyroidism. 1) The rate of parathyroidectomy has been found to dramatically decrease after introduction of cinacalcet. In fact, patients with tertiary hyperparathyroidism requiring surgical intervention are rare. Surgery is necessary for only $0.5 \%$ to $1 \%$ of patients with tertiary hyperparathyroidism after successful kidney transplantation. ${ }^{5)}$

The surgical indications for tertiary hyperparathyroidism and the timing of the operation remain controversial. The indications for parathyroidectomy in patients with tertiary hyperparathyroidism include persistent hypercalcemia more than 6 months after kidney transplant, low bone-mineral density, renal stone or nephrocalcinosis, deterioration of kidney graft resulting from tertiary hyperparathyroidism, symptomatic tertiary hyperparathyroidism such as bone and joint pain, bone fracture, pruritus, fatigue, depression, irritability, sleeplessness, peptic ulcer, etc., and enlarged parathyroid gland detected by ultrasound. ${ }^{6)}$

Recently, we performed a subtotal parathyroidectomy in a patient with tertiary hyperparathyroidism after kidney transplantation. We report this case and review the literature.

\section{Case Report}

A 47-year-old male presented with hypercalcemia and chronic fatigue. He had a history of chronic kidney disease due to immunoglobulin A ( $\lg A)$ nephropathy since 1978 and underwent kidney transplantation in 1998. After transplantation, his blood urea nitrogen (BUN) and creatinine levels were continuously high and the patient was considered to be experiencing chronic rejection. Seven years later, he started continuous ambulatory peritoneal dialysis (CAPD). He underwent another kidney transplantation in 2014. There was no rejection after the second kidney transplantation. However, his levels of calcium and parathyroid hormone $(\mathrm{PTH})$ remained high until now, four years
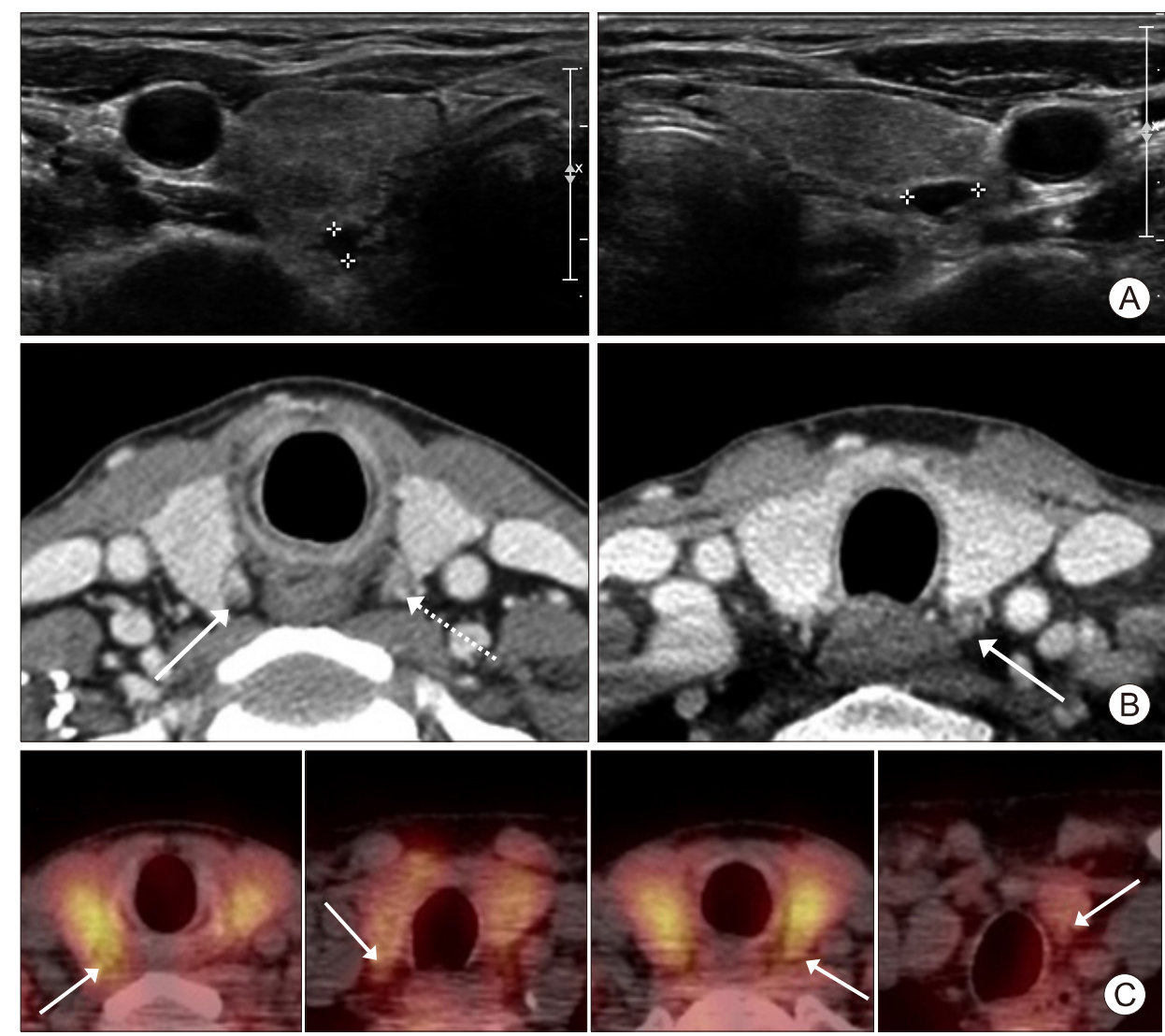

Fig. 1. Preoperative imaging studies. (A) Ultrasonography showing two cystic lesions in the right and left superior parathyroid glands. (B) Computed tomography scan showing three hyperplastic parathyroid glands at the right superior (solid line arrows), left superior (dash line arrows), and left inferior parathyroid glands. (C) Tc-99m methoxy iso butyl isonitrile (MIBI) parathyroid scan showing increased uptake in four parathyroid glands (solid line arrows indicates right superior parathyroid gland, right inferior parathyroid gland, left superior parathyroid gland, and left inferior parathyroid gland, in the order of the figures). 


\section{Subtotal Parathyroidectomy for Tertiary Hyperparathyroidism}

after transplantation.

Nephrologists sent him to us for consultation regarding a surgical intervention. There were no specific findings on physical examination. The vocal fold movement was intact on flexible laryngoscopic examination. His BUN/creatinine level was 14.7/1.04 (BUN normal range $8-20 \mathrm{mg} / \mathrm{dL}$, creatinine normal range $0.67-$ $1.17 \mathrm{mg} / \mathrm{dL})$, within the normal range. The intact PTH concentration was $130 \mathrm{pg} / \mathrm{mL}$ (normal range 15-65 $\mathrm{pg} / \mathrm{mL}$ ) and his calcium level was $11 \mathrm{mg} / \mathrm{dL}$ (normal range $8.6-10.3 \mathrm{mg} / \mathrm{dL}$ ). The patient's alkaline phosphatase and phosphate levels were $135 \mathrm{U} / \mathrm{L}$ (normal range $30-120 \mathrm{U}$ ), and $2.2 \mathrm{mg} / \mathrm{dL}$ (normal range $2.5-4.5 \mathrm{mg} / \mathrm{dL})$, respectively.

Two hypertrophied parathyroid glands were detected in the right and left superior parathyroid glands by neck ultrasonography (Fig. 1A) and three parathyroid glands by computed tomography (Fig. 1B) except for the right inferior parathyroid gland. There was increased uptake in four parathyroid glands on Tc-99m methoxy isobutyl isonitrile (MIBI) parathyroid scan with regional single photon emission computed tomography (SPECT) and no ectopic glands were identified (Fig. 1C).

A subtotal parathyroidectomy was performed. During the operation, four ovoid-shaped parathyroid glands measuring approximately $1 \mathrm{~cm}$ were identified. Three and half of these parathyroid glands were removed and a half of the smallest right inferior parathyroid gland was preserved in situ (Fig. 2). Frozen section biopsies of all parathyroid tissues were consistent with parathyroid hyperplasia. The permanent biopsy results were also the same. There were no perioperative complications and the postoperative course was uneventful.

The intact PTH level was reduced to $95.6 \mathrm{pg} / \mathrm{mL}$ and the calcium concentration was reduced to 9.9 $\mathrm{mg} / \mathrm{dL} 1$ day postoperatively. After three months, the
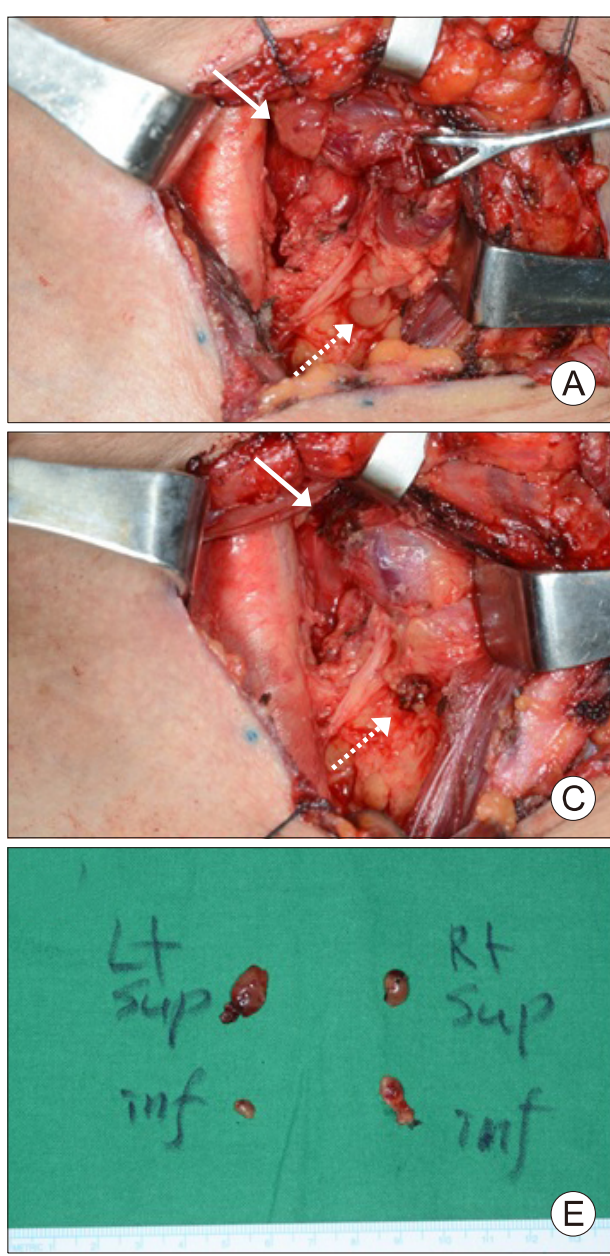
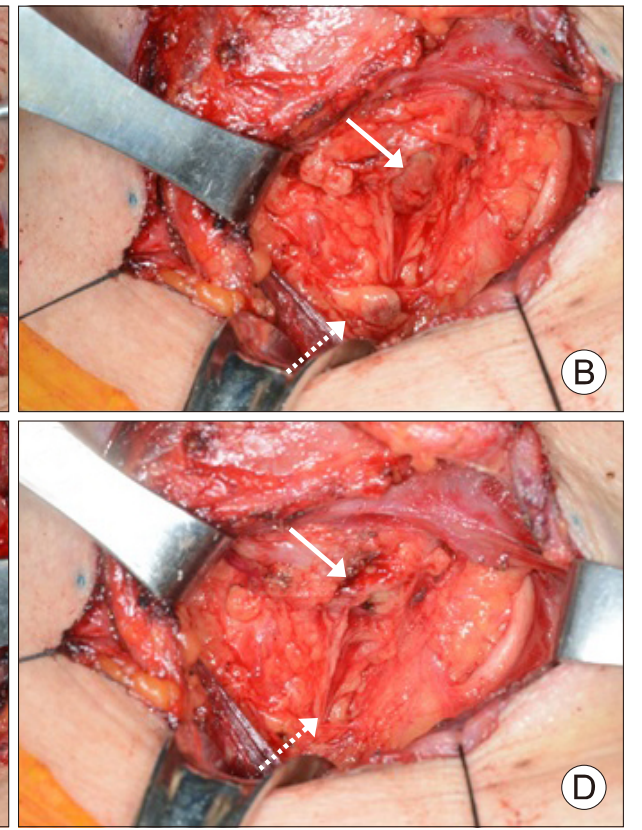

(D)
Fig. 2. Operative views of subtotal parathyroidectomy. (A) The right superior (solid line arrow) and right inferior parathyroid glands (dashed line arrow) are shown. (B) The left superior (solid line arrow) and left inferior parathyroid gland (dashed line arrow) are shown. (C) The right superior parathyroid gland (solid line arrow) and $1 / 2$ of right inferior parathyroid gland were removed with preservation of half of the right inferior parathyroid gland in situ (dashed line arrow). (D) The left superior (solid line arrow) and left inferior parathyroid gland (dashed line arrow) are removed. (E) Surgical specimen of the parathyroid glands. 
intact PTH level was $139 \mathrm{pg} / \mathrm{mL}$, slightly higher than normal range $(15-65 \mathrm{pg} / \mathrm{mL})$, and the calcium concentration was within normal range (10.3 mg/dL).

\section{Discussion}

Most patients with tertiary hyperparathyroidism are asymptomatic. However, they may experience bone pain or fractures, pruritus, nephrolithiasis, pancreatitis, soft tissue or vascular calcification, and mental status change. ${ }^{7)}$ In contrast to secondary hyperparathyroidism, laboratory investigation reveals hypercalcemia, hypophosphatemia, a moderately elevated PTH level, and usually an increased alkaline phosphatase level.

Imaging studies such as ultrasonography, neck computer tomography, neck magnetic resolution imaging, and parathyroid scan are not mandatory for diagnosis. However, preoperative imaging may be useful for localization of parathyroid glands and identification of existing ectopic parathyroid glands or supernumerary glands.

Although a calcimimetic agent is effective even in patients with severe hyperparathyroidism, medical treatment for tertiary hyperparathyroidism is not curative in some patients and surgery is the mainstay for patients with tertiary hyperparathyroidism. Subtotal parathyroidectomy and total parathyroidectomy with autotransplantation are widely accepted for tertiary hyperparathyroidism. Total parathyroidectomy without autograft is not an accepted option for patients with tertiary hyperparathyroidism because of the risk of persistent uncontrollable hypocalcemia after parathyroidectomy.

In total parathyroidectomy with autograft, all four glands are removed. The most normal-appearing gland is minced into $1-\mathrm{mm}^{3}$ pieces and transplanted into well-vascularized musculature such as the brachioradialis or sternocleidomastoid muscles. The implanted site is tagged by metallic clips or non-absorbable sutures. Subtotal parathyroidectomy includes removal of $31 / 2$ parathyroid glands, leaving 40-80 mg of gland marked with a metal clip or non-absorbable sutures.

Evidence is lacking regarding which procedure is most appropriate for tertiary hyperparathyroidism. The incidence of recurrence or hypoparathyroidism does not statistically differ between subtotal parathyroidectomy and total parathyroidectomy with autograft. ${ }^{9)}$ However, subtotal parathyroidectomy deteriorates kidney function less than total parathyroidectomy with autograft due to the postoperatively less pronounced transient hypoparathyroidism. ${ }^{10)}$ Therefore, most surgeons prefer subtotal parathyroidectomy for patients with tertiary hyperparathyroidism and successful kidney transplantation and recommend leaving a volume equivalent to four normal parathyroid glands to prevent hypoparathyroidism and hypocalcemia. Subtotal parathyroidectomy may be better to preserve kidney function after kidney transplantation.

Some authors recommend a limited parathyroidectomy less than a subtotal resection for tertiary hyperparathyroidism to avoid postoperative hypocalcemia. However, its efficacy has not yet been well determined. $^{11)}$

The definition of success after parathyroidectomy varies. Most articles define it as normal serum calcium levels at six months postoperatively. However, several articles have defined success as a normal PTH level and symptom improvement. ${ }^{12)}$ The success rates after surgery in tertiary hyperparathyroidism are high. Up to $74-94 \%$ of patients can be considered to be cured based on total serum calcium level after subtotal parathyroidectomy.' In our case, the calcium level was normal at 1 day and 3 months after surgery, while PTH level was normal at 1 day after surgery and slightly high at 3 months postoperatively. Now, 4 months after surgery, the surgical outcome of our case appeared to be a success based on calcium level. However, long-term follow-up is necessary to confirm a success of this case. It is a limitation of this study.

In conclusion, parathyroidectomy is the main treatment for tertiary hyperparathyroidism that does not respond to medical treatment, and subtotal parathyroidectomy is also a good surgical option for tertiary hyperparathyroidism. 
Subtotal Parathyroidectomy for Tertiary Hyperparathyroidism

\section{References}

1) Yang RL, Freeman K, Reinke CE, Fraker DL, Karakousis GC, Kelz RR, et al. Tertiary hyperparathyroidism in kidney transplant recipients: characteristics of patients selected for different treatment strategies. Transplantation 2012;94(1):70-6.

2) Jamal SA, Miller PD. Secondary and tertiary hyperparathyroidism. J Clin Densitom 2013;16(1):64-8.

3) Tominaga Y. Surgical and medical management of tertiary hyperparathyroidism. World J Endocrine Surg 2010;2(3):105-9.

4) Nichols G Jr, Roth SI. Case records of the Massachusetts General Hospital: Weekly clinicopathological exercises. Case 29-1963 Chronic renal disease and hypercalcemia. $N$ Engl J Med 1963;268:943-53.

5) Park JH, Kang SW, Jeong JJ, Nam KH, Chang HS, Chung $\mathrm{WY}$, et al. Surgical treatment of tertiary hyperparathyroidism after renal transplantation: a 31-year experience in a single institution. Endocr J 2011;58(10):827-33.

6) Randolph GW. Surgery of the thyroid and parathyroid glands. 2nd ed. Philadelphia, PA: Saunders, Elsevier; 2013.
7) Kerby JD, Rue LW, Blair H, Hudson S, Sellers MT, Diethelm AG. Operative treatment of tertiary hyperparathyroidism: a single-center experience. Ann Surg 1998;227(6):878-86.

8) Hsieh TM, Sun CK, Chen YT, Chou FF. Total parathyroidectomy versus subtotal parathyroidectomy in the treatment of tertiary hyperparathyroidism. Am Surg 2012;78(5):600-6.

9) Pitt SC, Sippel RS, Chen H. Secondary and tertiary hyperparathyroidism, state of the art surgical management. Surg Clin North Am 2009;89(5):1227-39.

10) Schlosser K, Endres N, Celik I, Fendrich V, Rothmund M, Fernandez ED. Surgical treatment of tertiary hyperparathyroidism: the choice of procedure matters! World J Surg 2007;31(10): 1947-53.

11) Pitt SC, Panneerselvan R, Chen H, Sippel RS. Tertiary hyperparathyroidism: is less than a subtotal resection ever appropriate? A study of long-term outcomes. Surgery 2009; 146(6):1130-7.

12) Tang JA, Friedman J, Hwang MS, Salapatas AM, Bonzelaar LB, Friedman M. Parathyroidectomy for tertiary hyperparathyroidism: a systematic review. Am J Otolaryngenol 2017;38(5): 630-5. 